\title{
EL AUMENTO DE LA EXPRESIÓN DEL ARNm DE LA ENZIMA CONVERTIDORA DE ANGIOTENSINA I HOMÓLOGA (ECA-2) INDUCIDO POR ATORVASTATINA SE ASOCIA A MENOR FIBROSIS E HIPERTROFIA VENTRICULAR IZQUIERDA EN UN MODELO DE CARDIOMIOPATÍA DIABÉTICA
}

\author{
Cristian Aguilar ${ }^{1, a}$, Freddy Ventura ${ }^{2, b}$, Luis Rodríguez-Delfín ${ }^{1,3,4, c}$
}

\begin{abstract}
RESUMEN
Objetivos. Evaluar el efecto de atorvastatina sobre la progresión del remodelado cardiaco y la expresión de ECA-2 en el miocardio de ratas diabéticas. Materiales y métodos. La diabetes fue inducida en ratas Holtzman con una inyección intraperitoneal de estreptozotocina. Los animales fueron divididos en tres grupos: (1) ratas control, (2) ratas diabéticas y (3) ratas diabéticas tratadas con atorvastatina (50 mg/kg/día). Después de ocho semanas de tratamiento, los corazones fueron extraídos para el análisis morfométrico, la cuantificación de colágeno y la determinación de los niveles de ARNm de ECA y ECA-2. Resultados. El índice de hipertrofia ventricular y el depósito de colágeno se incrementaron significativamente en las ratas diabéticas. La administración de atorvastatina previno estos cambios sin modificar los niveles de colesterol. La hiperglicemia produjo un incremento significativo en los niveles del ARNm de ECA y una marcada disminución en la expresión de ECA-2 en el miocardio de ratas diabéticas. La administración de atorvastatina indujo la expresión del ARNm de ECA-2 e inhibió la sobreexpresión del ARNm de ECA en el miocardio de las ratas diabéticas. Conclusiones. Nuestros resultados indican que la atorvastatina, independientemente de su capacidad para disminuir el colesterol, normaliza la relación de la expresión de ECA/ECA-2 y atenúa el desarrollo del remodelado adverso en el corazón diabético.
\end{abstract}

Palabras clave: Miocardiopatías; Sistema renina-angiotensina; Reacción en cadena de la polimerasa de transcriptasa inversa (Fuente: DeCS BIREME).

\section{ATORVASTATIN INDUCED INCREASE IN HOMOLOGOUS ANGIOTENSIN I CONVERTING ENZYME (ACE2) mRNA IS ASSOCIATED TO DECREASED FIBROSIS AND DECREASED LEFT VENTRICULAR HYPERTROPHY IN A RAT MODEL OF DIABETIC CARDIOMYOPATHY}

\begin{abstract}
Objectives. This study has investigated the effect of atorvastatin on the progression of cardiac remodelling and ACE2 expression in diabetic myocardium in rats. Materials and Methods. Diabetes was induced in Holtzman rats with an intraperitoneal injection of streptozotocin. The animals were divided into 3 groups: (1) normal control rats, (2) diabetic rats and (3) diabetic rats treated orally with atorvastatin $(50 \mathrm{mg} / \mathrm{kg} / \mathrm{day})$. After eight weeks of treatment, the hearts were removed for morphometric studies, collagen content assay and genetic expressions of ACE and ACE2 mRNA. Results. Myocardial hypertrophy index and collagen deposition were increased in diabetic rats, but not in the treated-diabetic rats, without producing changes in cholesterol levels. Myocardial ACE mRNA levels were increased while ACE2 mRNA levels were decreased in diabetic rats. Atorvastatin administration attenuated overexpression of ACE mRNA and overexpression of ACE-2 mRNA in diabetic rats. Conclusions. Our results indicate that atorvastatin, independently of its cholesterol-lowering capacity, lowers the ACE/ACE2 ratio to normal values and attenuates the development of adverse remodeling in the diabetic heart.
\end{abstract}

Key words: Cardiomyopathies; Renin-angiotensin system; Reverse transcriptase polymerace chain reaction (source: $\mathrm{MeSH}$ NLM)

\footnotetext{
Departamento de Anatomía Patológica, Hospital Nacional Edgardo Rebagliati Martins. Lima, Perú.

Departamento de Ciencias Básicas, Facultad de Medicina, Universidad Nacional de Trujillo. Trujillo, Perú

Laboratorio de Biología Molecular, Facultad de Biología, Universidad Nacional Pedro Ruiz Gallo. Chiclayo, Perú.

"Investigación y Diagnóstico en Genética y Biología Molecular "GEN MOL". Trujillo, Perú.

a Médico, Residente de Anatomía Patológica, HNERM; ' ${ }^{b}$ Químico farmacéutico, Magíster en Fisiología y Biofísica; ' Biólogo, Doctor en Ciencias, área de Genética.
} 


\section{INTRODUCCIÓN}

Las enfermedades cardiovasculares son las principales causas de morbimortalidad en pacientes diabéticos (1). Durante las últimas tres décadas diversos estudios epidemiológicos, clínicos y experimentales han confirmado la existencia de una miocardiopatía asociada a la diabetes ${ }^{(2)}$. Esta condición clínica se define actualmente como la presencia de disfunción miocárdica en ausencia de hipertensión y enfermedad arterial coronaria (1). La hipertrofia y la fibrosis del miocardio constituyen los principales cambios patológicos observados en la miocardiopatía diabética, inicialmente estos cambios contribuirán a la disfunción diastólica del ventrículo izquierdo (VI), posteriormente a la disfunción sistólica y finalmente al desarrollo de insuficiencia cardíaca (IC) ${ }^{(2-4)}$.

El remodelado cardíaco es un evento central tanto en el desarrollo como en la progresión de la IC ${ }^{(2)}$. Evidencias clínicas y experimentales sugieren que la activación del sistema renina-angiotensina (SRA) y la generación de la angiotensina (Ang) II cumplen una función crítica en el remodelado estructural y funcional en el corazón diabético ${ }^{(4,5)}$. Además, es bien sabido que el bloqueo del SRA, tanto en la diabetes clínica como experimental, puede atenuar la progresión y, en algunos casos incluso, revertir cambios estructurales en el corazón diabético ${ }^{(6)}$.

Durante décadas, la enzima convertidora de angiotensina (ECA) se consideró una enzima clave en el SRA. Sin embargo, esta visión clásica del SRA ha sido cuestionada por el descubrimiento de la enzima convertidora I homóloga (ECA-2) ${ }^{(7)}$. De hecho, recientemente se ha descubierto que los niveles de Ang II dependen no solo de las actividades de la renina y de la ECA, sino que también de la ECA-2 ${ }^{(8)}$. Esta enzima degrada Ang I a Ang-(1-9) y Ang II a Ang- (1-7), por lo tanto, antagoniza la acción de la $\mathrm{ECA}^{(7,8)}$. Así, las acciones vasoconstrictoras/proliferativas o vasodilatadoras/antiproliferativas del SRA en los tejidos estarían reguladas por el balance de ECA/ECA-2.

Existe evidencia experimental que demuestra el significado funcional de ECA-2 en la patofisiología cardiovascular ${ }^{(8)}$. Al respecto, reportes recientes han señalado que los animales knockout para ECA-2 muestran un aumento de Ang II y alteraciones en la contractibilidad cardíaca, mientras que ratones que sobreexpresan el gen de la ECA-2 muestran menores cifras de presión arterial sistólica ${ }^{(9)}$. Estas observaciones permiten proponer que la inhibición de la ECA-2 podría aumentar la enfermedad cardiovascular y que su mayor expresión podría producir un efecto benéfico.

Las estatinas, inhibidoras de la 3-hidroxi-3-metilglutaril coenzima A (HMG-CoA) reductasa, han demostrado clínicamente reducir la morbilidad y mortalidad cardiovascular ${ }^{(10)}$. Aunque los beneficios de las estatinas habían sido inicialmente atribuidos a su efecto hipolipemiante, estudios recientes han propuesto que las estatinas actuarían directamente a través de otros mecanismos celulares ${ }^{(11)}$. Recientemente se ha demostrado que la inhibición de la via Rho-cinasa por parte de las estatinas atenúa el remodelado cardiaco inducido por Ang II ${ }^{(12)}$. Estas observaciones sugieren que las estatinas podrían inhibir el remodelado cardiaco al interferir con la señalización de Ang II en el corazón diabético. El objetivo de nuestro estudio fue evaluar el efecto de la administración de atorvastatina sobre la progresión del remodelado cardiaco y la expresión de ECA-2 en el miocardio de ratas en ausencia y en presencia de diabetes.

\section{MATERIALES Y MÉTODOS}

\section{MODELO ANIMAL DE DIABETES EXPERIMENTAL}

El protocolo experimental de este estudio se realizó de acuerdo con la Guía para el cuidado y uso de animales de laboratorio, publicado por el National Health Institute (NIH Publication $\left.N^{\circ} 85-23,1996\right)$. La diabetes fue inducida en ratas macho Holtzman (200-220 g) procedente de la Universidad Nacional Agraria La Molina, con una sola inyección intraperitoneal de estreptozotocina (STZ) $50 \mathrm{mg} / \mathrm{kg}$ preparado en $0,1 \mathrm{~mol} / \mathrm{L}$ de buffer citrato $(\mathrm{pH}$ 4,5 ). Solamente las ratas con niveles de glucosa sérica mayor a $200 \mathrm{mg} / \mathrm{dL}$ tres días después de la inyección de STZ fueron incluidas en el estudio ${ }^{(3)}$. Las ratas control fueron inyectadas con el mismo volumen de buffer citrato solo.

\section{PROTOCOLO Y GRUPOS EXPERIMENTALES}

Las ratas fueron divididas en tres grupos: (1) ratas control normales $(C, n=8),(2)$ ratas diabéticas $(D, n=8)$ y $(3)$ ratas diabéticas tratadas con atorvastatina $(\mathrm{DA}, \mathrm{n}=8)$. La atorvastatina se administra por gavaje en una dosis de $50 \mathrm{mg} /$ $\mathrm{kg} /$ día durante ocho semanas. La dosis de estatinas utilizada en el presente estudio se basó en un estudio previo ${ }^{(13)}$ en el que se demostró que una dosis de $50 \mathrm{mg} / \mathrm{kg}$ de atorvastatina fue suficiente para bloquear RhoA/Rho en los corazones de ratas. Asimismo, se ha demostrado que esta dosis produce concentraciones plasmáticas comparables a las obtenidas tras la administración oral de dosis clínicas de atorvastatina en el hombre ${ }^{(14)}$.

Los animales fueron mantenidos bajo condiciones estándar de iluminación (ciclo luz-oscuridad de 12 h) y alimentados con una dieta estándar y agua ad libitum. Al final de las ocho semanas, los animales fueron anestesiados mediante inyección intraperitoneal de 
pentobarbital sódico (40 mg/kg). Después de recoger la sangre, el corazón fue extraído rápidamente. En el músculo papilar, el corazón se dividió en dos partes en sentido transversal: la porción distal para el estudio histomorfométrico y la porción proximal para la determinación de colágeno y de la expresión génica.

\section{DE LAS DETERMINACIONES SÉRICAS}

Al final del estudio, las ratas fueron sometidas a un ayuno de $12 \mathrm{~h}$, luego se tomaron muestras de sangre por punción cardiaca. El suero fue separado y, posteriormente, se analizaron los niveles de glucosa y colesterol por colorimetría (Biochemical diagnostic kit, Human, Wiesbaden, Germany) mediante un espectrofotómetro UV/Vis (Jenway 6505 UVIVIS, Jenway, Felsted, UK).

\section{HISTOLOGÍA Y ANÁLISIS MORFOMÉTRICO}

Bajo anestesia se procedió a extraer los corazones, posteriormente fueron lavados con solución salina, secados y pesados. El índice de hipertrofia del VI fue calculado por la relación peso $\mathrm{VI}$ / peso corporal (el tabique intraventricular fue incluido en el peso del VI). Finalmente, el VI fue fijado en formaldehído al $10 \%$, incluida en parafina, cortado en láminas de $4-\mu \mathrm{m}$ de espesor y teñido con hematoxilina-eosina y solución tricrómica de Masson.

Con la ayuda de un computador equipado con un software para análisis de imagen (ImageJ, NIH, Bethesda, USA) el área de la sección transversal de los cardiomiocitos fue medida para ser utilizada como un indicador del tamaño celular. En cada animal se midió al menos 50 cardiomiocitos, cortados transversalmente y con su núcleo claramente en posición central ${ }^{(15)}$. Por otra parte, el grado de fibrosis perivascular se estimó por la relación entre el colágeno perivascular y el área luminal de las arterias coronarias intramiocárdicas en un total de diez campos por corazón. Un solo investigador, desconocedor de la naturaleza de los grupos experimentales, estuvo a cargo del análisis histológico.

\section{DETERMINACIÓN DEL ARNm DE LA ECA Y ECA-2 POR TRANSCRIPCIÓN REVERSA DE LA REACCIÓN DE LA CADENA DE POLIMERASA (RT-PCR)}

Para determinar si la transcripción de la ECA y ECA-2 en el miocardio se vio afectada por la diabetes, la expresión del ARNm de la ECA y ECA-2 se evaluó mediante transcripción reversa acoplada a la reacción en cadena de ADN polimerasa (RT-PCR). El ARN total fue extraído del miocardio utilizando el kit PureLink ${ }^{\text {TM }}$ Micro-to-Midi Total RNA Purification kit (Invitrogen, Carlsbad, CA, USA) de acuerdo con la recomendación del fabricante.
Se empleó diez microlitros de ARN total para sintetizar la cDNA usando el kit SuperScript III First-Strand Synthesis kit (Invitrogen, Carlsbad, CA, USA). El cDNA fue amplificado por PCR utilizando mediante Platinum ${ }^{2}$ Taq DNA Polymerase (Invitrogen, Carlsbad, CA, USA) para la ECA, ECA-2 y gliceraldehído 3 fosfato deshidrogenasa (GA$\mathrm{DPH})$ como el control, respectivamente. Las secuencias de cebadores fueron las siguientes: para ECA: forward, 5'-CAC CGG CAA GGT CTG CTT-3', reverse, 5'-CTT GGC ATA GTT TCG TGA GGAA-3'; para ECA-2: forward 5'-GTG CAC AAA GGT CAC AAT GG-3', reverse, 5'-TGT TTC ATC ATG AGG CAG AGG-3'; para GAPDH: forward, 5'-CCC TCA AGA TTG TCA GCA ATGC-3', reverse, 5'GTC CTC AGT GTA GCC CAG GAT-3'. Las condiciones de amplificación fueron: 30 ciclos de desnaturalización a $94{ }^{\circ} \mathrm{C}$ durante $1 \mathrm{~min}$, hibridación a $62{ }^{\circ} \mathrm{C}$ durante $1 \mathrm{~min}$, y elongación a $72{ }^{\circ} \mathrm{C}$ durante 1 min para ECA; 38 ciclos de desnaturalización a $94{ }^{\circ} \mathrm{C}$ durante $1 \mathrm{~min}$, hibridación a $60{ }^{\circ} \mathrm{C}$ durante $1 \mathrm{~min}$, y elongación a $72{ }^{\circ} \mathrm{C}$ durante 1 min para ECA-2; y 35 ciclos de desnaturalización a $94{ }^{\circ} \mathrm{C}$ durante $1 \mathrm{~min}$, hibridación a $62^{\circ} \mathrm{C}$ durante $1 \mathrm{~min}$, y elongación a $72^{\circ} \mathrm{C}$ durante 1 min para GAPDH.

La intensidad de las bandas se cuantificó por densitometría mediante un planímetro digital (ImageJ, NIH, Bethesda, USA); y se normalizó con respecto a la banda del gen control GADPH, con expresión estándar en el miocardio, en un corrido electroforético en gel de agarosa.

\section{DETERMINACIÓN DEL CONTENIDO DE COLÁGENO}

La determinación del contenido en colágeno ventricular se realizó mediante un método bioquímico basado en la cuantificación de hidroxiprolina (HOP) originalmente descrita por Reddy ${ }^{(16)}$, con modificaciones menores. Brevemente, el tejido (50 mg) fue homogeneizado manualmente en agua destilada. Los homogeneizados se hidrolizaron en un volumen de $\mathrm{NaOH} 4 \mathrm{~N}$ a $120^{\circ} \mathrm{C}$ durante $20 \mathrm{~min}$. Las muestras se oxidaron con $450 \mu \mathrm{L}$ de reactivo de cloramina $\mathrm{T}$ durante $25 \mathrm{~min}$ a temperatura ambiente. Las muestras se mezclaron luego con $500 \mu \mathrm{L}$ de reactivo de Erlich e incubados a $65{ }^{\circ} \mathrm{C}$ durante 20 min. La absorbancia de la muestra fue leída a $550 \mathrm{~nm}$ en un espectrofotómetro (Jenway 6505 UV/VIS, Jenway, Felsted, UK). El contenido de colágeno fue estimado a partir de una curva de calibración $(0-10 \mu \mathrm{g} / \mathrm{ml}$ de la Lhidroxiprolina), suponiendo que el contenido de HOP representa el $12,5 \%$ del tejido colágeno.

\section{ANÁLISIS ESTADÍSTICO}

Los resultados fueron expresados como promedio + error estándar. Se realizó un análisis de varianza (ANOVA) para establecer diferencias entre grupos, seguidos 
Tabla 1. Efecto de atorvastatina sobre las variables morfométricas y metabólicas

\begin{tabular}{lccc}
\hline Variable & $\begin{array}{c}\text { Control } \\
(\mathbf{n}=8)\end{array}$ & $\begin{array}{c}\text { Diabético } \\
(\mathbf{n}=8)\end{array}$ & $\begin{array}{c}\text { Diabético-ATV } \\
(\mathbf{n}=8)\end{array}$ \\
\hline PC $(\mathbf{g})$ & $371,39 \pm 14,25$ & $247,36 \pm 7,91^{*}$ & $255,53 \pm 10,26^{*}$ \\
PVI/PC (mg/g) & $2,11 \pm 0,04$ & $2,59 \pm 0,11^{*}$ & $2,23 \pm 0,09^{*+}$ \\
Glucosa (mg/dL) & $123,5 \pm 16,02$ & $417,75 \pm 21,12^{*}$ & $408,25 \pm 21,95^{*}$ \\
Colesterol (mg/dL) & $86,39 \pm 5,58$ & $107,24 \pm 2,88^{*}$ & $104,46 \pm 2,98^{*}$ \\
\hline
\end{tabular}

PC: peso corporal; PVI: peso del ventrículo izquierdo. Los valores se expresan como Media \pm SD. * $p<0,05$ vs. grupo control; ${ }^{\dagger} p<0,05$ vs. grupo diabético (ANOVA seguido por la prueba de comparaciones múltiples de Tukey).

de la postprueba de Tukey para establecer diferencias individuales. Se consideró una diferencia estadísticamente significativa al valor de $p<0,05$. Los datos fueron procesados con el paquete estadístico SPSS 15.0.

\section{RESULTADOS}

\section{PARÁMETROS METABÓLICOS}

A lo largo del período de ocho semanas de estudio, las ratas diabéticas mostraron pérdida de peso corporal, polifagia y polidipsia. El tratamiento con atorvastatina no evitó la pérdida de peso ni redujo el consumo elevado de comida y agua en las ratas diabéticas.

Las ratas diabéticas presentaron un marcado incremento de la glucosa sérica en comparación con las ratas control $(417,75 \pm 21,12 \mathrm{mg} / \mathrm{dL}$ vs $123,55 \pm 16,02$ $\mathrm{mg} / \mathrm{dL} ; \mathrm{p}<0,05)$. El tratamiento de las ratas diabéticas con atorvastatina no modificó los niveles de glucosa $(408,25 \pm 21,95 \mathrm{mg} / \mathrm{dL})$ (Tabla 1). Estos datos sugieren que la atorvastatina no ejerció un efecto metabólico significativo en el control glucémico de estos animales.

Los animales diabéticos presentaron niveles más elevados de colesterol sérico en comparación con los animales control $(107,24 \pm 2,88 \mathrm{mg} / \mathrm{dL}$ vs $86,39 \pm 5,58 \mathrm{mg} / \mathrm{dL}$; $p<0,05)$. El tratamiento con atorvastatina, no afectó significativamente los niveles de lípidos en las ratas diabéticas tratadas $(104,46 \pm 2,98 \mathrm{mg} / \mathrm{dl})$. Nuestros datos muestran que la atorvastatina no afectó los niveles de colesterol sérico en los animales tratados, lo que nos permite investigar in vivo los efectos pleiotrópicos de la atorvastatina.

\section{HIPERTROFIA CARDÍACA}

El peso del VI con relación al peso corporal como medida del índice de hipertrofia del VI fue significativamente mayor en las ratas diabéticas $(2,59 \pm 0,11)$, en comparación con las ratas control $(2,11 \pm 0,04 ; p<0,05)$. El tratamiento con atorvastatina atenuó significativamente el desarrollo de hipertrofia del VI en las ratas diabéticas con respecto a las no tratadas $(2,23 \pm 0,09$ vs $2,59 \pm 0,11$; $\mathrm{p}<0,05)$ (Tabla 1).
El examen histológico reveló que la hiperglicemia también provocó un incremento significativo en el área transversal de los cardiomiocitos de las ratas diabéticas $\left(308,11 \pm 27,51 \mu \mathrm{m}^{2}\right)$, en comparación con las ratas control $\left(176 \pm 12,61 \mu \mathrm{m}^{2} ; \mathrm{p}<0,05\right)$. El tratamiento con atorvastatina redujo el aumento en el área transversal de los cardiomiocitos en ratas diabéticas $(217,9 \pm 23,41$ $\left.\mu \mathrm{m}^{2} ; \mathrm{p}<0,05\right)$ (Figura 1).

\section{EL CONTENIDO DE COLÁGENO}

EIVIdelasratas diabéticasseencontrósubstancialmente fibrosado, con un marcado incremento en el depósito de colágeno tanto intersticial como perivascular en comparación con las ratas control. El tratamiento con atorvastatina fue capaz de normalizar el incremento de la fibrosis perivascular e intersticial en las ratas diabéticas (Figura 2a-f). El análisis histológico demostró que la fibrosis perivascular afecta principalmente

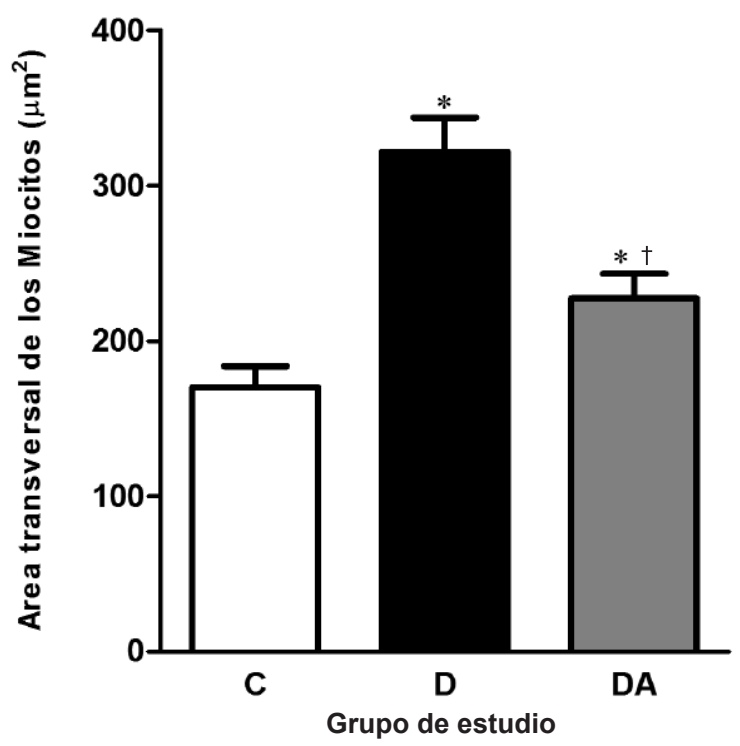

Figura 1. Área transversal de los cardiomiocitos en el ventrículo izquierdo de los tres grupos experimentales.

Cuantificación del área transversal de los cardiomiocitos en el grupo control (C), grupo diabético (D) y grupo diabético-atorvastatina (DA). Los datos se muestran como Media $\pm S D$. ${ }^{*} p<0,05$ vs grupo control; ${ }^{\dagger} p<0,05$ vs grupo diabético. 

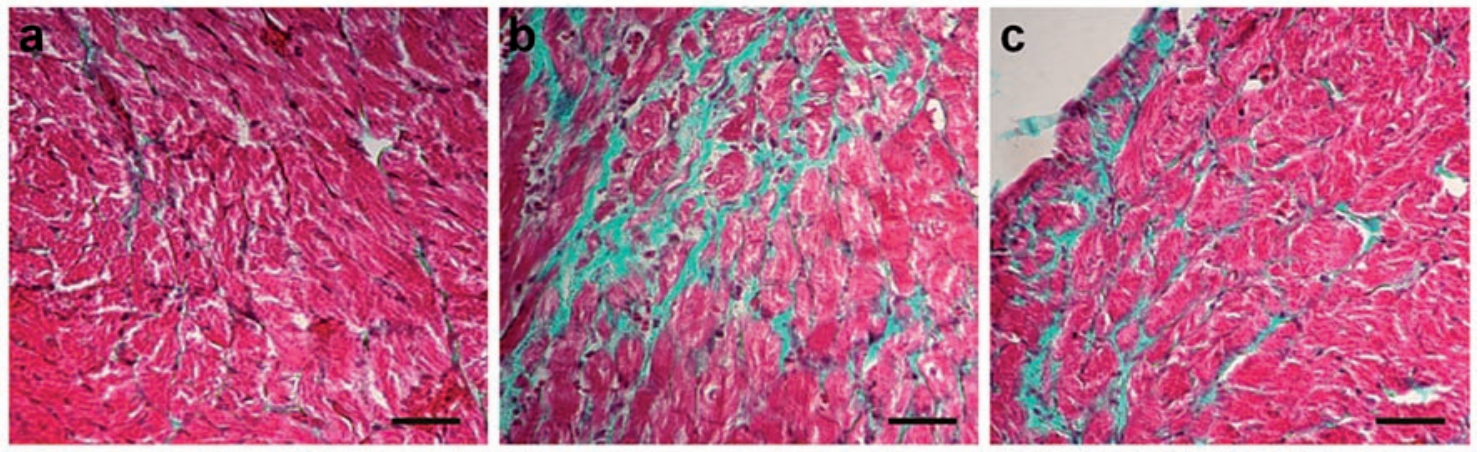

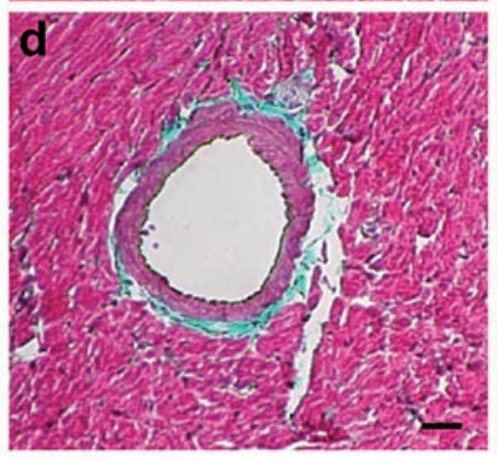

g

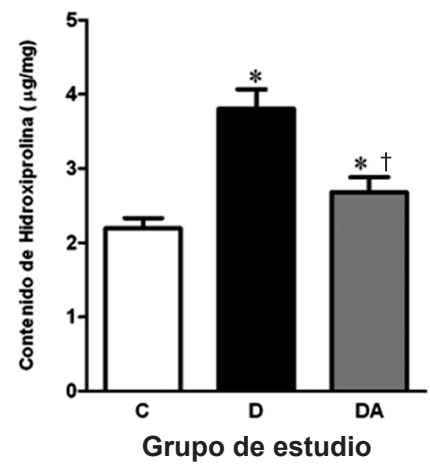

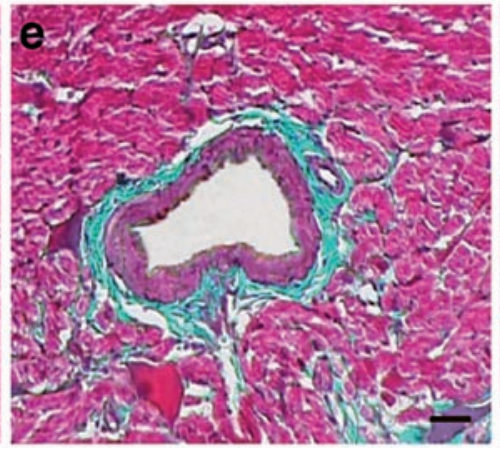

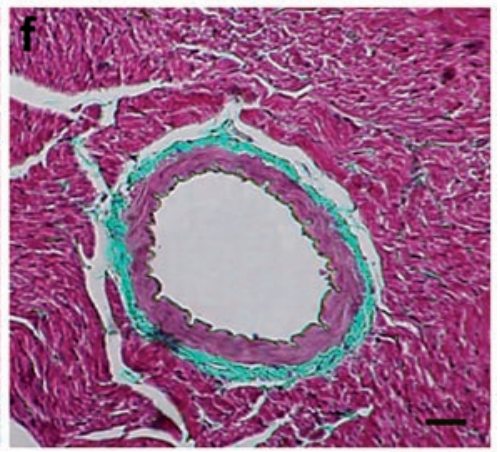

h

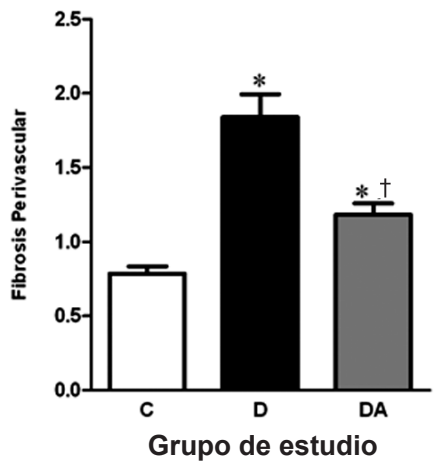

Figura 2. Fibrosis intersticial y perivascular en el ventrículo izquierdo de los tres grupos experimentales.

Fibrosis intersticial en el VI de las ratas del (a) grupo control, (b) grupo diabético o (c) grupo diabético-atorvastatina. Fibrosis perivascular en las ratas del (d) grupo control, (e) grupo diabético o (f) grupo diabético-atorvastatina. Cortes coloreados con el método Tricrómica de Masson (100x). (g) Cuantificación del contenido de hidroxiprolina en el VI (ug/mg). (h) Cuantificación del área de fibrosis perivascular del VI. Los datos se muestran como Media \pm SD. ${ }^{*} p<0,05$ vs. grupo control; ${ }^{\dagger} p<0,05$ vs. grupo diabético.

a las arterias intramiocárdicas de pequeño calibre (Figura 2h). Las extensas áreas de fibrosis intersticial se ubicaron, predominantemente, en el endomisio y perimisio.

Una medida bioquímica de la fibrosis cardiaca, el contenido de hidroxiprolina, también se encontró significativamente incrementada en el VI de las ratas diabéticas, en comparación con las ratas control $(3,92 \pm 0,57 \mu \mathrm{g} / \mathrm{mg}$ vs $2,16 \pm 0,09 \mu \mathrm{g} / \mathrm{mg} ; p<0,05)$. La atorvastatina redujo significativamente el incremento del contenido de hidroxiprolina en el VI de las ratas diabéticas $(2,78 \pm 0,26 \mu \mathrm{g} / \mathrm{mg}$ vs $3,92 \pm 0,57 \mu g / m g ; p<0,05$ ) (Fig. 2i).

\section{EXPRESIÓN GÉNICA DE LA ECA Y ECA-2}

El ARNm de la ECA en el tejido cardíaco de las ratas diabéticas fue marcadamente mayor a la observada en las ratas control $(3,56 \pm 0,8$ vs $1,36 \pm 0,2 ; p<0,05)$. El incremento del ARNm de la ECA fue significativamente atenuado por atorvastatina en las ratas diabéticas $(2,09 \pm 0,3$ vs $3,56 \pm 0,8 ; p<0,05)$. Por el contrario, la expresión del ARNm de ECA-2 disminuyó significativamente en el miocardio de las ratas diabéticas, en comparación con el grupo control $(1,17 \pm 0,6$ vs $1,78 \pm 0,2 ; p<0,05)$. La administración de atorvastatina incrementó significativamente los niveles del ARNm de la ECA-2 en comparación con las ratas 
diabéticas sin tratamiento $(3,06 \pm 0,3$ vs $1,17 \pm 0,6 ; p<0,05)$. Esta diferencia se acentuó aun más al representarse como un ratio entre los niveles del ARNm de ECA/ECA-2 para cada animal (Figura 3).

\section{DISCUSIÓN}

El tratamiento con estatinas ha demostrado que tiene un papel significativo en la mejora del remodelado cardiaco en diversos modelos de daño cardiaco. Este trabajo demuestra, por primera vez, que la atorvastatina, utilizada en una dosis demasiado baja como para reducir los niveles de colesterol, atenúa el desarrollo de las alteraciones estructurales del remodelado cardíaco y normaliza la relación entre la expresión génica de la ECA/ECA-2 en un modelo experimental de miocardiopatía diabética.

La rata representa un excelente modelo para el estudio de los "efectos pleiotrópicos" de las estatinas, puesto que su perfil lipídico ha demostrado ser refractario al tratamiento con estatinas (17). En el presente estudio, los niveles séricos de colesterol fueron marcadamente mayores en las ratas diabéticas, sin embargo, la administración de atorvastatina no modificó este parámetro. Del mismo modo, el tratamiento de las ratas diabéticas con atorvastatina durante 12 semanas no modificó los niveles de glucosa (Tabla 1). Estos datos sugieren que la administración de atorvastatina no ejerció efecto metabólico importante en relación al control glucémico y lipídico de nuestros animales.

La activación patológica del SRA con el consiguiente incremento de la Ang II local produce efectos nocivos sobre el miocardio durante el remodelado cardiaco ${ }^{(5)}$. La diabetes per se ha demostrado ser capaz de activar el SRA cardiaco favoreciendo el incremento de la densidad de los receptores AT1 y actividad de la ECA en el miocardio ${ }^{(2,5)}$. Asimismo, el tratamiento con drogas que interrumpen la cascada de SRA ha demostrado atenuar la hipertrofia cardiaca inducida por Ang II (6). Nuestros hallazgos demuestran que la administración de atorvastatina reduce el aumento del área transversal de los cardiomiocitos y el incremento en el peso del VI en las ratas diabéticas.

En 1999, Luo (18) fue el primero en demostrar que el tratamiento con una estatina (simvastatina) reduce la actividad de la ECA y la cantidad de Ang II en el miocardio de ratas con estenosis aórtica. El efecto antihipertrófico de las estatinas podría deberse, en parte, a la disminución de la actividad de la ECA (18) o la expresión de los receptores AT1 (19) en el miocardio. Sin embargo, la capacidad de las estatinas para inhibir
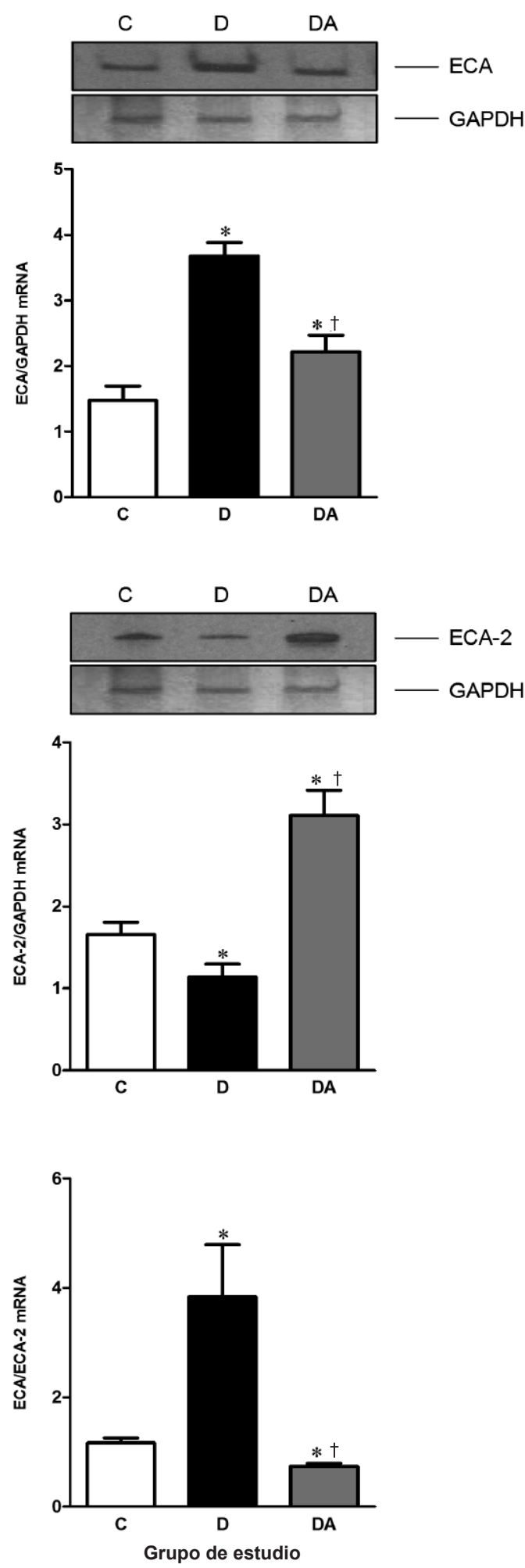

Figura 3. Expresión del ARNm de ECA y ECA-2 en el ventrículo izquierdo de los tres grupos experimentales.

ECA miocárdica (panel superior), ECA-2 (panel medio) y ratio ECA/ ECA-2 (calculado por cada animal) (panel inferior) en el grupo control (C), grupo diabético (D) y grupo diabético-atorvastatina (DA). Los datos se muestran como Media \pm SD. ${ }^{*} p<0,05$ vs. grupo control; ${ }^{\dagger} p<0,05$ vs. grupo diabético. 
la hipertrofia cardiaca en respuesta a otros agonistas, como la noradrenalina ${ }^{(20)}$ y endotelina-1 (21), sugieren que el mecanismo predominante de acción incluye mecanismos distales a los receptores AT1. Esta hipótesis se apoya en los hallazgos de Ocaranza (22), quien demostró que RhoA-cinasa previene el bloqueo del remodelado vascular inducido por Ang II en mayor medida que el bloqueo de los receptores AT1.

Numerosos estudios experimentales y clínicos han demostrado que la fibrosis miocárdica es el sustrato patológico de la miocardiopatía diabética ${ }^{(23)}$. Dependiendo de su distribución y cantidad, la fibrosis del miocardio puede aumentar desfavorablemente la rigidez del VI disminuyendo dramáticamente la distensibilidad miocárdica, favoreciendo el desarrollo de IC ${ }^{(24)}$. Es bien sabido que la diabetes por sí sola es capaz de iniciar la expresión de genes profibróticos y el remodelado de la matriz extracelular (MEC) en el miocardio ${ }^{(5)}$. De hecho, se ha demostrado que la alteración en el Ilenado diastólico del VI se asocia con cambios recíprocos en el perfil de expresión génica del colágeno así como su depósito y distribución en el VI de ratas diabéticas ${ }^{(24,25)}$.

Nuestros resultados muestran que la diabetes provoca un incremento en el depósito del contenido de colágeno intramiocárdico, con predominio de fibrosis subendocárdica y perivascular en las ratas diabéticas (Figura 2). La administración de atorvastatina disminuyó significativamente el depósito de colágeno en el intersticio y en el espacio perivascular en las ratas diabéticas. Nuestros resultados son consistentes con trabajos previos, que muestran que las estatinas son capaces de reducir el depósito de colágeno inducido por Ang II al disminuir la expresión del ARNm del TGF- $\beta$ en fibroblastos cardia$\cos { }^{(26)}$. Estas observaciones permiten asumir que las estatinas disminuyen la fibrosis miocárdica inducida por Ang II, sugiriendo un claro efecto antifibrótico de las estatinas en el corazón diabético.

Desde la década pasada la Ang II se ha propuesto como uno de los mediadores más importantes del remodelado cardiaco que precede a la IC, independientemente de la injuria inicial ${ }^{(5)}$. Recientemente, una vía paralela del SRA ha sido descubierta ${ }^{(7)}$ donde la ECA-2 emerge como un novedoso antagonista de los efectos deletéreos que ejerce la Ang II. Aunque la ECA-2 hidroliza varios sustratos, la producción de Ang-(1-7) parece tener el rol más importante a nivel cardiovascular. La Ang(1-7) ha mostrado ser un importante regulador de la función cardiovascular promoviendo la vasodilatación y un efecto antiproliferativo ${ }^{(8)}$. Por lo tanto, la ECA-2 intervendría en la mantención de la homeostasis cardiovascular, contrarregulando los efectos nocivos derivados de la $\operatorname{ECA}^{(7,8)}$.
La Ang II a través de los receptores AT1 induce cambios estructurales en el miocardio, incluyendo la acumulación de proteínas de la MEC y la inducción de factores de crecimiento profibróticos ${ }^{(5,25)}$. En la presente investigación, el incremento en el contenido de colágeno en las ratas diabéticas se acompaño de un marcado incremento en el ratio de ECA/ECA-2 en el miocardio, sugiriendo un incremento local de Ang II. Nuestros datos son consistentes con los de Landau ${ }^{(25)}$, quien ha demostrado que la diabetes provoca una disminución progresiva en la expresión de ECA-2 mientras que favorece un marcado incremento tanto en la expresión como en la actividad de ECA en el miocardio de ratas diabéticas. Qin ${ }^{27)}$, en ratas mutantes para el alelo nulo del gen CCK-A (colecistoquinina), reportó que la disminución de la hipertrofia cardiaca producida por atorvastatina en un modelo distinto, de sobrecarga de presión, se asocia con una reducción en la expresión de ECA-2 y proteína; nuestros resultados sugieren que la capacidad de atorvastatina para atenuar la progresión del remodelado cardíaco inducido por la Ang II se asocia con la disminución de la expresión de la ECA y un progresivo incremento en la expresión de la ECA-2 en el corazón diabético.

La vía de señalización intracelular RhoA-cinasa es un novel mecanismo descubierto en la última década, con una participación destacada en el remodelado cardiovascular (11). La relación entre la via RhoA-cinasa y el SRA ha sido evaluada por Aoki ${ }^{28)}$ quien demostró que la Ang II regula la expresión de diversos genes profibróticos a través de la activación de la via RhoA-cinasa. Al respecto, se ha reportado recientemente que en las ratas sometidas a infusión continua de Ang II, la inhibición de la via RhoA-cinasa previene por sí sola el incremento de la expresión del TFG- $\beta$ y el depósito de la MEC en la aorta ${ }^{(29)}$. Nuestros resultados son consistentes con los de Ocaranza ${ }^{(30)}$, quien ha reportado que la inhibición de la vía RhoA-cinasa con fasudil favorece el incremento de la expresión de ECA-2 en aorta de rata, mientras que disminuye la expresión génica y la actividad de ECA. Colectivamente, nuestros resultados permiten proponer que las estatinas atenúan la expresión de genes profibróticos inducidos por Ang II a través de la inhibición de la activación de RhoA-cinasa.

En conclusión, la activación no balanceada del SRA sugiere al marcado incremento de los efectos de Ang II en el miocardio diabético como un posible mecanismo que contribuye al remodelado cardiaco. Nuestros resultados sugieren que atorvastatina, independientemente de su capacidad para disminuir el colesterol, promueve el bloqueo de las vías de señalización que activan la remodelación cardiaca en la diabetes mediante el aumento del ARNm ECA-2 cardíaco. Así, nuestros datos sugieren que la atorvastatina seria una eficaz $y$ 
novedosa estrategia para prevenir la progresión de la remodelación cardiaca en la miocardiopatía diabética.

\section{Contribución de Autoría}

CA participó en la concepción y diseño del trabajo, análisis e interpretación de datos y redacción del manuscrito. FV participó en el diseño del trabajo y análisis bioquímico de las muestras. LR-D participó en el diseño del trabajo y las pruebas de RT-PCR, interpretación y redacción parcial del manuscrito. Todos revisaron y aprobaron la versión final del trabajo.

\section{Fuentes de Financiamiento}

Autofinanciado.

\section{Conflictos de Interés}

Los autores declaran no tener conflictos de interés en la publicación del presente artículo.

\section{REFERENCIAS BIBLIOGRÁFICAS}

1. Hayat S, Patel B, Khattar R, Malik R. Diabetic cardiomyopathy: mechanisms, diagnosis and treatment. Clin Sci. 2004;107:539-57.

2. Adeghate E. Molecular and cellular basis of the etiology and management of diabetic cardiomyopathy: a short review. Mol Cell Biochem. 2004;261:187-91.

3. Akula A, Kota M, Gopisetty S, Chitrapu R, Kalagara $\mathbf{M}$, Kalagara $\mathbf{S}$, et al. Biochemical, histological and echocardiographic changes during experimental cardiomyopathy in STZ-induced diabetic rats. Pharmacol Res. 2003;48:429-35.

4. Asbun J, Villarreal F. The pathogenesis of myocardial fibrosis in the setting of diabetic cardiomyopathy. J Am Coll Cardiol. 2006;47:693-700.

5. De Mello WC. Impaired cell communication in the diabetic heart. The role of the renin angiotensin system. Mol Cell Biochem. 2007;296:53-8.

6. Tsutsui $H$, Matsushima $S$, Kinugawa $S$, Ide $T$, Inoue $\mathbf{N}$, Ohta Y, et al. Angiotensin II type 1 receptor blocker attenuates myocardial remodeling and preserves diastolic function in diabetic heart. Hypertens Res. 2007;30:439-49.

7. Donoghue M, Hsieh F, Baronas E, Godbout K, Gosselin $\mathbf{M}$, Stagliano N, et al. A novel angiotensin-converting enzyme-related carboxypeptidase (ACE2) converts angiotensin I to angiotensin 1-9. Circ Res. 2000;87:E1-9.

8. Crackower M, Sarao R, Oudit G, Yagil C, Kozieradzki I, Scanga S, et al. Angiotensin-converting enzyme 2 is an essential regulator of heart function. Nature. 2002;417:822-8.

9. Donoghue M, Wakimoto $\mathrm{H}$, Maguire $\mathrm{C}$, Acton $\mathrm{S}$, Hales $\mathrm{P}$, Stagliano N, et al. Heart block, ventricular tachycardia, and suden death in ACE2 transgenic mice with downregulated connexins. J Mol Cell Cardiol. 2003;35:1043-53.

10. Kjekhus $\mathbf{J}$, Pedersen $\mathbf{T}$. The effects of simvastatin of the incidence of heart failure in patients with coronary heart disease. J Card Fail. 1997;3:249-54.
11. Rikitake Y, Liao J. Rho GTPases, statins and nitric oxide. Circ Res. 2005;97:1232-5.

12. Kang B, Mehta J. Rosuvastatin attenuates Ang II-mediated cardiomyocyte hypertrophy via inhibition of LOX-1. Mol Cardiovasc Pharmacol Ther. 2009;14(4):283-91.

13. Riad A, Du J, StiehI S, Westermann D, Mohr Z, Sobirey $\mathbf{M}$, et al. Low-dose treatment with atorvastatin leads to antioxidative and anti-inflammatory effects in diabetes mellitus. Eur J Pharmacol. 2007;569:204-11.

14. Chen G, Yao L, Lu X, Li L, Hu S. Tissue-specific effects of atorvastatin on 3-hydroxy-3-methylglutarylcoenzyme A reductase expression and activity in spontaneously hypertensive rats. Acta Pharmacol Sin. 2008;29(10):1181-6.

15. Matsubara L, Narikawa S, Ferreira N, Zornoff L, Matsubara B. Myocardial Remodeling in Chronic Pressure or Volume Overload in the Rat Heart. Arq Bras Cardiol. 2006;86(2):126-30.

16. Reddy G, Enweka C. A simplified method for the analysis of hydroxyproline in biological tissues. Clin Biochem. 1996;29:225-9.

17. Lopez I, Relaño E, Herrera E, Bocos S. Estudio del mecanismo de acción hipolipemiante de la lovastatina en la rata. Clin Invest Arterioscl. 2003;15(6):248-57.

18. Luo J, Zhang W, Zhang G, Guan J, Chen X. Simvastatin inhibits cardiac hypertrophy and angiotensin-converting enzyme activity in rats with aortic stenosis. Clin Exp Pharmacol Phys. 1999;26:903-8.

19. Wassmann S, Laufs U, Bäumer A, Müller K, Konkol C, Sauer $\mathbf{H}$, et al. Inhibition of geranylgranylation reduces angiotensin II-mediated free radical production in vascular smooth muscle cells: involvement of angiotensin AT1 receptor expression and Rac1 GTPase. Mol Pharmacol. 2001;59:646-54.

20. Luo J, Xie F, Zhang W, Ma X, Guan J, Chen X. Simvastatin inhibits noradrenaline-induced hypertrophy of cultured neonatal rat cardiomyocytes. $\mathrm{Br} \mathrm{J}$ Pharmacol. 2001;132:159-64.

21. Hernández $O$, Pérez D, Navarro J, Sánchez R, Hernán$\operatorname{dez} \mathbf{G}$, Díaz C, et al. Effects of 3-hydroxy-3-methylglutarylCoA reductase inhibitors, atorvastatin and simvastatin, on the expression of endothelin-1 and endothelial nitric oxide synthase in vascular endothelial cells. J Clin Invest. 1998;101:2711-9.

22. Ocaranza $\mathbf{M}$, Rivera $\mathbf{P}$, Jalil J. Increased expression of homologous angiotensin I converting enzyme (ACE2) in the arterial wall of rats with genetically elevated levels of Angiotensin II: effects of candesartan and fasudil. Rev Chil Cardiol. 2008;27(2):173-82.

23. Riva E, Andreoni G, Bianchi R, Latini R, Luvara G, Jeremic $\mathbf{G}$, et al. Changes in diastolic function and collagen content in normotensive and hypertensive rats with longterm streptozotocin-induced diabetes. Pharmacol Res. 1998;37:233-40.

24. Pauschinger M, Knopf D, Petschauer S, Doerner A, PoIler W, Schwimmbeck $\mathbf{P}$, et al. Dilated cardiomyopathy is associated with significant changes in collagen type I/III ratio. Circulation. 1999;99:2750-6.

25. Landau D, Chayat C, Zucker N, Golomb E, Yagil C, Yagil Y, et al. Early blood pressure-independent cardiac changes in diabetic rats. J Endocrinol. 2008;197:75-83. 
26. Martin J, Denver R, Bailey M, Krum H. In vitro inhibitory effects of atorvastatin on cardiac fibroblasts: implications for ventricular remodelling. Clin Exp Pharmacol Physiol. 2005;32(9):697-701.

27. Qin X, Jia C, Pan M, Shen A, Jing H. Effect of atorvastatin on ACE2 expression in pressure overload induced cardiac hypertrophy in rats. Zhong Nan Da Xue Xue Bao Yi Xue Ban. 2008;33(5):438-42.

28. Aoki H, Izumo S, Sadoshima J. Angiotensin II activates RhoA in cardiac myocytes: a critical role of RhoA in angiotensin II-induced premyofibril formation. Circ Res. 1998;82:666-76.

29. Takeda K, Ichiki T, Tokunou T, lino N, Fujii S, Kitabatake A, et al. Critical role of Rho-kinase and MEK/ERK pathways for angiotensin II-induced plasminogen activator inhibitor type-1 gene expression. Arterioscler Thromb Vasc Biol. 2001;21:868-73.
30. Ocaranza M, Rivera P, Jalil J. Inhibition of Rho-kynase decreases systolic blood pressure and activates the vasodilating pathway of the renin angiotensin system in the arterial wall of hypertensive rats. 2008;27(1):65-76.
Correspondencia: Luis Rodríguez Delfín

Dirección: Laboratorio de Biología Molecular

Facultad de Ciencias Biológicas Medicina, Universidad Nacional de Pedro Ruiz Gallo. Lambayeque, Perú.

Teléfono: (51) 044 31-0210/ (51) 94 88-55160

Correo electrónico: ladelfin@amauta.rcp.net.pe

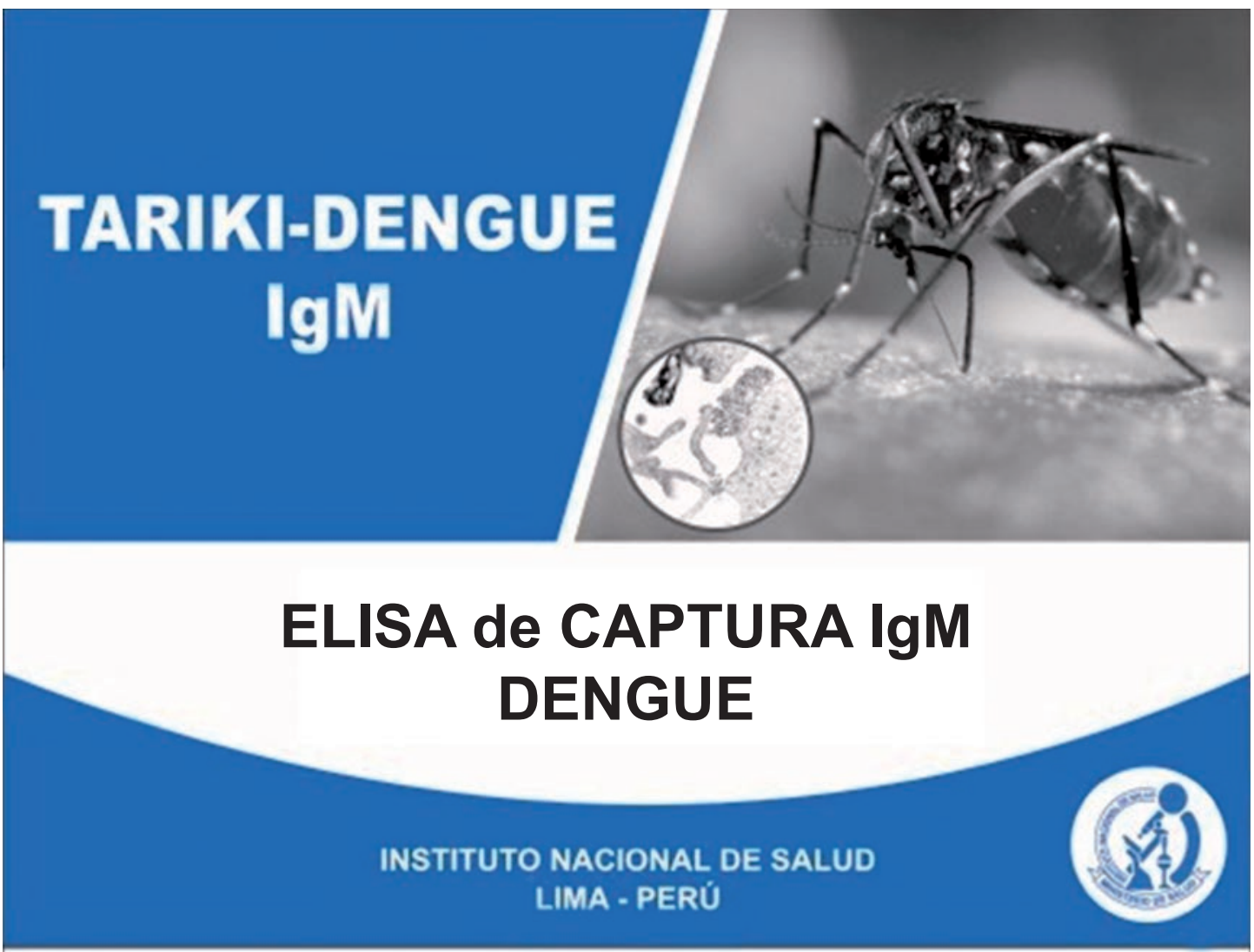

\section{Pronto disponible en el Perú....}

\section{Kit para la determinación de anticuerpos IgM anti Dengue, desarrollado y producido por el Instituto Nacional de Salud. INS/MINSA}

